\title{
An improved method for quantitative sugar analysis of glycoproteins
}

\author{
Soohyun Kim ${ }^{1,3}$, Seung II Kim ${ }^{1}$, Kwon-Soo Ha ${ }^{1}$ \\ and Sun-Hee Leem ${ }^{2}$ \\ ${ }^{1}$ Biomolecule Research Team, Korea Basic Science Institute, \\ Taejon 305-333, Korea \\ ${ }^{2}$ Department of Biology, Dong-A University, Pusan 604-714, Korea \\ ${ }^{3}$ Corresponding author: Tel, +82-42-865-3425; \\ Fax, +82-42-865-3419; E-mail, shkim@comp.kbsi.re.kr
}

Accepted 19 September 2000

Abbreviations: Fuc, fucose; GalN, galactosamine; GlcN, glucosamine; Gal, galactose; Glc, glucose; Man, mannose; GalNAc, $N$ acetylgalactosamine; GIcNAc, $\mathrm{N}$-acetylglucosamine; TFA, trifluoroacetic acid; HPAEC-PAD, high-pH anion exchange chromatography with pulsed amperometric detection; PVDF, polyvinylidene difluoride; EPO, erythropoietin.

\begin{abstract}
Although there are numerous methods available to hydrolyze glycans utilizing strong acids, it all requires lengthy steps to obtain quantitative yield. We have developed a new simple one-step method for analysis of amino and neutral monosaccharides of glycoproteins quantitatively. Free monosaccharides were found to be stable during hydrolysis of glycans with $6 \mathrm{~N} \mathrm{HCl}$ at $80^{\circ} \mathrm{C}$ up to $2 \mathrm{~h}$. Using this condition, analysis of free monosaccharides hydrolyzed from the bovine fetuin showed sugar composition of Gal : Man : GIcN : GalN = $13.2: 11.0: 15.5: 2.6$, which is closely matched with the reported value of 12.4 : 9.6 : 17.2 : 2.7 (Townsend et al., ABRF News 8: 14, 1997). This method was shown to be applicable to varieties of well-characterized glycoproteins, erythropoietin, fibrinogen and soybean agglutinin. The amounts of sugars released under the condition were very close to the experimental values by other procedures or to the theoretical ones. This condition was found to be suitable for direct sugar analysis of fetuin, which have been immobilized onto polyvinylidene difluoride membrane. Based on these results, it support that the $6 \mathrm{~N} \mathrm{HCl} / 80^{\circ} \mathrm{C} / 2 \mathrm{~h}$ is the simplest method for quantitative analysis of monosaccharide composition of glycoproteins.
\end{abstract}

Keywords: simultaneous analysis, sugar composition, glycoprotein

\section{Introduction}

Glycosylation is a major post-translational modification found on cytoplasmic, secreted, and membrane proteins (Green et al., 1988; Ha-Lee et al., 2000). Oligosaccharides occupy a large part of glycoproteins and can modify the structure, dynamics and functional activities of their conjugate polypeptides. The glycosylation of a protein is cell type-specific (Kornfeld and Kornfeld, 1985) and is influenced by extra-cellular environment and the method of cell culture, especially for recombinant glycoproteins (Spellman, 1990). Thus, it is very important to characterize the structure of glycans in order to fully understand the structural basis of a glycoprotein's function.

Quantitative monosaccharide analysis of a given glycoprotein provides the molar ratio of individual sugars to protein and provides information regarding types of oligosaccharide ( $\mathrm{N}$ - or O-glycans), the basis to design a structural elucidation strategy, and a measure for production consistency of recombinant glycoprotein therapeutics (Spellman, 1990) such as erythropoietin (EPO). Sugar composition of a glycoprotein is obtained through serial steps of cleavage of all glycosidic bonds, separation, detection and quantification of the released sugars. At present, the most widely used method is the acid hydrolysis followed by quantitative analysis with high-pH anion exchange chromatography with pulsed amperometric detection (HPAEC-PAD) (Townsend et al., 1997). Glycoproteins have been digested with two different acids separately to determine amino and neutral sugars, since each kind of monosaccharides had different hydrolyzing yields and stabilities against acids (Fan et al., 1994). The amounts of amino sugars are often determined with strong acid hydrolysis using $\mathrm{HCl}$ or sulfuric acid, while neutral sugars are underestimated because of their unstability. Mild hydrolysis by using TFA is good to maintain integrity of amino and even neutral sugars, but often insufficient to release sugars completely. Because of the shortcoming in each condition, two kinds of hydrolyses should have been commonly used to determine sugar composition with an imperfection.

Unless rigorous protocols are followed for preparation of standards and sample by weight, inaccuracies may occur. Considerable variability ( $\pm 25-30 \%$ ) among sugar analysis often observed, and a part of the source of variability was inter-laboratory errors (Townsend et al., 1997). When monosaccharide standards were analyzed on the same instrument in several laboratories, the standard deviation was no greater than $15 \%$, suggest- 
ing that experimental error might be as high as $10-20 \%$. If we can get similar or greater results than the two hydrolyses method by one-step hydrolysis, it should be valuable for quantitative sugar analysis of glycoproteins.

\section{Materials and Methods}

\section{Materials}

All reagents were purchased from Sigma (St. Louis, MA) except followings: monosaccharide mixture standard from Dionex (Sunnyvale, CA), polyvinylidene difluoride (PVDF) membrane from Millipore (Bedford, MA) and $50 \% \mathrm{NaOH}$ from Fisher (Pittsburgh, PA).

\section{Monosaccharide stability against acid media}

Free monosaccharide mixture containing $0.5 \mathrm{nmol}$ of each fucose (Fuc), galactosamine (GalN), glucosamine (GlcN), galactose (Gal), glucose (Glc) and mannose (Man) was incubated with $6 \mathrm{M} \mathrm{HCl}$ or $2 \mathrm{M}$ trifluoroacetic acid (TFA) at 80 or $100^{\circ} \mathrm{C}$. An aliquot was removed from the incubations at 1, 2, 4 and $6 \mathrm{~h}$, respectively. The aliquots were dried, dissolved in distilled $\mathrm{H}_{2} \mathrm{O}\left(\mathrm{dH}_{2} \mathrm{O}\right)$ and redried to obtain acid free sugar residues.

\section{Hydrolysis of glycoproteins}

The hydrolysis of glycoproteins was carried out in the same manner as described above. The hydrolyzates were evaporated to dryness using a SpeedVac (Sarvant Instruments Inc., Holbrook, NY), resuspended in $\mathrm{dH}_{2} \mathrm{O}$, and then processed using HPAEC-PAD.

Procedures for $10 \%$ SDS-PAGE and transferring to PVDF membrane of proteins were described elsewhere (Weitzhandler et al., 1993). Bands containing blotted proteins were cut, washed briefly with $\mathrm{dH}_{2} \mathrm{O}$, and hydrolyzed with the same procedures as above.

\section{HPAEC-PAD analysis}

The amount of monosaccharides was measured by using a Bio-LC (DX-300, Dionex Corp., Sunnyvale, CA) equipped with a CarboPac PA1 column $(4 \times 250 \mathrm{~mm}$, Dionex $)$ and a guard column $(4 \times 50 \mathrm{~mm})$. Monosaccharides were separated with $16 \mathrm{mM} \mathrm{NaOH}$ at a flow rate of $1 \mathrm{ml} / \mathrm{min}$ and detected on a pulsed electrochemical detector (PED2) under the following settings: $E 1=0.05 \mathrm{~V}, \mathrm{t} 1$ $=400 \mathrm{~ms} ; \mathrm{E} 2=0.75 \mathrm{~V}, \mathrm{t} 2=200 \mathrm{~ms}$; and $\mathrm{E} 3=-0.15 \mathrm{~V}$, $\mathrm{t} 3=400 \mathrm{~ms}$. The column was regenerated with $0.2 \mathrm{M}$ $\mathrm{NaOH}$ between every injection. The data were analyzed with on-line chromatography software, $\mathrm{Al}-450$ (Dionex).

\section{Results}

\section{New hydrolysis method}

The stability of monosaccharides against acids was inve- stigated to find an optimal condition for simultaneous analysis of both amino and neutral sugars. Neutral oligosaccharides of glycoproteins are commonly consisted of five monosaccharides, Fuc, $\mathrm{N}$-acetylgalactosamine (GalNAc), $N$-acetylglucosamine (GlcNAc), Gal, and Man. Amino sugars, GalNAc and GlcNAc are converted to GalN and GlcN by de- $N$-acetylation during hydrolysis, respectively. In this study, Gal and GlcN as typical examples of neutral and amino sugars, respectively, were presented to simplify figures. Other sugars showed similar results (data not shown). Our study was carried out on the assumption that all glycans were in a de-sialylated form, and only amino and neutral sugars were compared each other, although many glycoprotein oligosaccharides were sialylated.

Under the condition of $6 \mathrm{M} \mathrm{HCl}$ at $100^{\circ} \mathrm{C}$, neutral

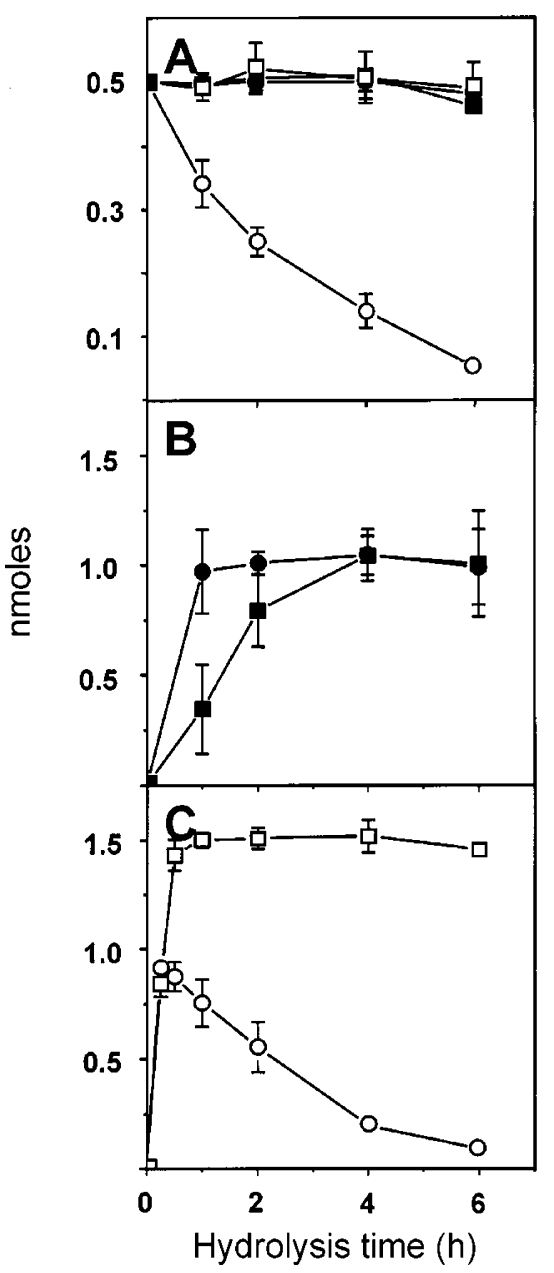

Figure 1. Acid Hydrolysis of bovine fetuin at $100^{\circ} \mathrm{C}$. A) A mixture of each $0.5 \mathrm{nmol}$ monosaccharides was incubated with $6 \mathrm{M} \mathrm{HCl}$ (open symbol) or 2 M TFA (closed symbol) to check stability. B) $3.5 \mu \mathrm{g}$ of fetuin was hydrolyzed with $2 \mathrm{M} \mathrm{TFA}$, or C) $6 \mathrm{M} \mathrm{HCl}$ for the indicated times. After hydrolysis, the hydrolyzates were analyzed with HPAEC as explained in the "Materials and Methods". Data are mean \pm S.D. from three independent experiments. $\bigcirc$, $\mathrm{Gal} ; \square, \mathrm{GlcN}$. 
sugars were decomposed very quickly, while amino sugars were stable at least up to $6 \mathrm{~h}$ (Figure 1A). $70 \%$ of the initial Gal amount was remained after $1 \mathrm{~h}$ incubation and $10 \%$ at $6 \mathrm{~h}$. These results suggested the hydrolysis in $6 \mathrm{M} \mathrm{HCl}$ is not suitable for neutral sugar analysis of glycoproteins. Monosaccharides were, however, resistant in $2 \mathrm{M} \mathrm{TFA}$ at $100^{\circ} \mathrm{C}$ (Figure $1 \mathrm{~A}$ ) during the incubation, suggesting this condition might be satisfactory for simultaneous analysis of both amino and neutral sugars.

The same condition with 2 M TFA was applied to hydrolyze bovine fetuin (Figure 1B). Fetuin has been used as a model system in many studies relating to the structure, biosynthesis, and functions of glycoconjugates because of availability of this protein and the substantial information about its carbohydrate units (Spiro and Bhoyroo, 1974; Edge and Spiro, 1987; Green et al., 1988). Fetuin has each three complex type of $N$ - and mucin type of O-glycans. Townsend et al. (1997) inferred a theoretical ratio of Gal : Man : GlcN : GalN = 12 : $9: 15: 3$ from the previously described informations, and observed a close value of $12.4: 9.6: 17.2: 2.7$. The little differences between the values might have resulted from characteristic heterogeneous biosynthesis of oligosaccharides with experimental errors. Hydrolysis for $4 \mathrm{~h}$ of fetuin with TFA produced a ratio of Gal : Man : GlcN : GalN = $12.1: 6.4: 12.1: 1.5$. Those were $20-50$ $\%$ lower than the theoretical or observed values except Gal, and the values were not improved with further incubation significantly. This result indicated that TFA hydrolysis of glycoproteins at $100^{\circ} \mathrm{C}$ was not enough to release all sugars from glycoproteins. Fan et al. (1994) also described incomplete digestion of glycoproteins with TFA. When fetuin was hydrolyzed with $6 \mathrm{M} \mathrm{HCl}$ at $100^{\circ} \mathrm{C}$, amino sugars were completely released after 2 $\mathrm{h}$ incubation, but neutral sugars were decomposed very quickly (Figure $1 C$ ), as expected in sugar stability test. The ratio of Gal : Man : GlcN : GalN was $6.3: 2.3: 17.3$ :2.6, which was quite different from the described values by Townsend et al. (1997). Those two incorrect compositional ratios suggested that hydrolysis with $\mathrm{HCl}$ or TFA at $100^{\circ} \mathrm{C}$ could not be used to supply correct information for oligosaccharide structure, but only to identify if a given protein was glycosylated. The combined ratio of the amino sugar values with $6 \mathrm{M} \mathrm{HCl}$ and the neutral sugar values with TFA at $100^{\circ} \mathrm{C}$ was Gal : Man : GlcN : GalN = $12.1: 6.4: 17.3: 2.6$. It was in agreement with the observed value (Townsend et al., 1997) except Man. It should be noted that they used shorter hydrolysis time ( $3 \mathrm{~h}$ ) compared to our condition ( $4 \mathrm{~h}$ ) for TFA hydrolysis. Man seemed to be relatively weaker than another neutral sugars, which was consistent with the result of Weitzhandler et al. (1993).

Since either TFA or $\mathrm{HCl}$ hydrolysis at high temperature did not achieve proper quantitative results for both amino and neutral monosaccharides, we lowered incubation temperature to $80^{\circ} \mathrm{C}$, which has been used for sialic acid analysis. In this case, it was expected that $\mathrm{HCl}$ could be a better media to release monosaccharides from glycoproteins, because TFA digested proteins incompletely. When free monosaccharides were incubated with $6 \mathrm{M} \mathrm{HCl}$ at $80^{\circ} \mathrm{C}$, they showed more than $98 \%$ stability up to $2 \mathrm{~h}$ although Gal decomposed slightly with further incubation (Figure 2A). $\mathrm{N}$-acetyl groups of amino sugars were also completely removed under the condition, which enabled complete separation from the neutral sugars (data not shown).

Hydrolysis of fetuin under $6 \mathrm{M} \mathrm{HCl}$ at $80^{\circ} \mathrm{C}$ gave the maximum yield of monosaccharides at $2 \mathrm{~h}$ (Figure 2B). As shown in Table 1, the calculated ratio of Gal : Man : GlcN : GalN was $13.2: 11.0: 15.5: 2.6$, which was very close to the observed value by Townsend et al. (1997).

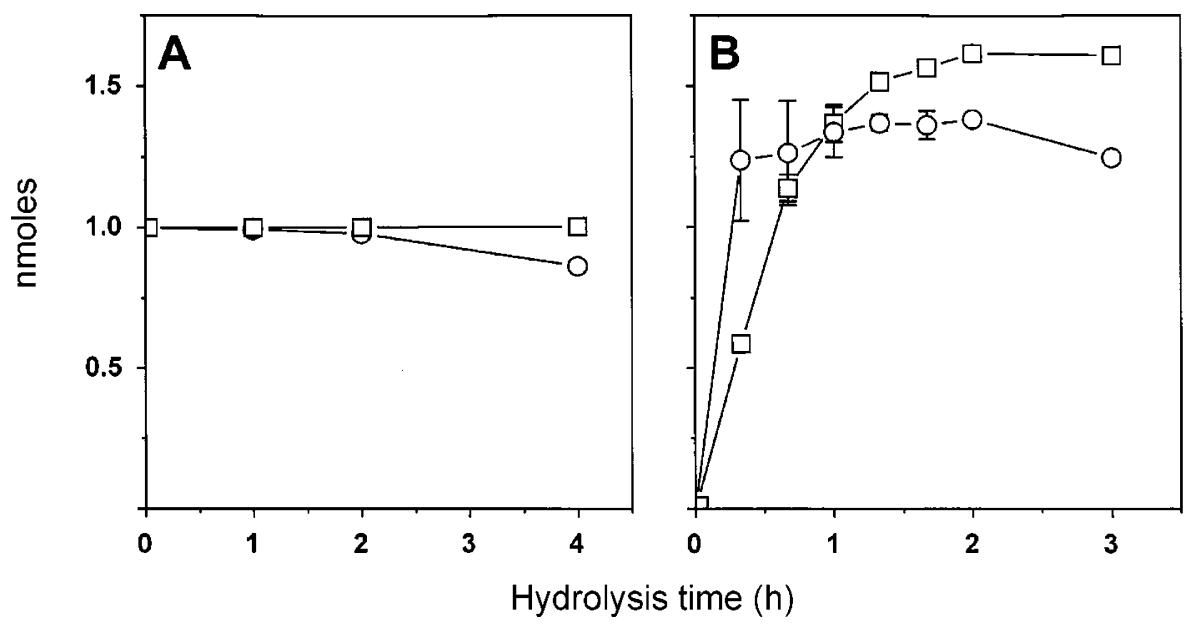

Figure 2. Acid Hydrolysis of bovine fetuin at $80^{\circ} \mathrm{C}$. (A) A mixture of each $1.0 \mathrm{nmol}$ monosaccharides was incubated with $6 \mathrm{M} \mathrm{HCl}$ to test stability. (B) $3.5 \mu \mathrm{g}$ of fetuin was hydrolyzed with $6 \mathrm{M} \mathrm{HCl}$ for the indicated times. The hydrolyzates were analyzed as described in Figure 1. Data are mean \pm S.D. from three independent experiments. $\bigcirc, \mathrm{Gal} ; \square, \mathrm{GlcN}$. 
Table 1. Summary of the acid hydrolyses of bovine fetuin under various conditions. Fetuin $(3.5 \mu \mathrm{g})$ was taken for hydrolysis. The data are the average from at least three different experiments

\begin{tabular}{lrrrc}
\hline \multirow{2}{*}{ Conditions } & \multicolumn{4}{c}{ Observed (mol sugar/mol protein) } \\
\cline { 2 - 5 } & \multicolumn{1}{c}{ Gal } & Man & GlcN & GalN \\
\hline Townsend et al. $(1997)^{\mathrm{a}}$ & 12.4 & 9.6 & 17.2 & 2.7 \\
$6 \mathrm{M} \mathrm{HCl} / 80^{\circ} \mathrm{C} / 2 \mathrm{~h}$ & 13.2 & 11.0 & 15.5 & 2.6 \\
$6 \mathrm{M} \mathrm{HCl} / 100^{\circ} \mathrm{C} / 2 \mathrm{~h}$ & 6.3 & 2.3 & 17.3 & 2.6 \\
$2 \mathrm{M} \mathrm{TFA} / 100^{\circ} \mathrm{C} / 4 \mathrm{~h}$ & 12.1 & 6.4 & 12.1 & 1.5 \\
PVDF $^{\mathrm{b}}$ & 9.5 & 6.9 & 12.1 & 2.3 \\
& $(12.5)^{\mathrm{c}}$ & $(9.1)$ & $(15.9)$ & $(3.0)$ \\
\hline
\end{tabular}

${ }^{a} 4 \mathrm{M} \mathrm{HCl}$ for amino and $2 \mathrm{M}$ TFA for neutral sugars at $100^{\circ} \mathrm{C}$ for $3 \mathrm{~h}$.

${ }^{b} 3.5 \mu \mathrm{g}$ of fetuin was separated on $10 \%$ SDS-PAGE, transferred to a PVDF membrane, and hydrolyzed under the condition of $6 \mathrm{M} \mathrm{HCl}$ at $80^{\circ} \mathrm{C}$ for $2 \mathrm{~h}$.

${ }^{c}$ Values in parenthesis are corrected ones by a factor of 1.32 .

The errors (2-15\%) were acceptable considering regular experimental error range (10-20\%) for monosaccharide analysis. As described, quantitative sugar analysis makes it possible not only to estimate molar ratio of monosaccharides to a protein but also to predict oligosaccharide structures. The value of GalN suggested partial site occupancy of $3 \mathrm{O}$-linked sites. The number of Man and GICN residues including error was in agreement with $3 \mathrm{~N}$-linked sites being fully occupied with either bior tri-antennary type structures. In addition, Gal residues accounted for the three multi-antennary structures and three mucin type $O$-linked glycans.

\section{Monosaccharides analysis of another glycoproteins}

To verify the efficiency of our new hydrolysis condition, we digested another glycoprotein, EPO known to have the same complex type and mucin type oligosaccharides for the sites as fetuin, but with high sugar contents (Lee et al., 1996). The present hydrolysis condition produced again similar numbers of monosaccharides to the ones by regular methods from EPO (Table 2). Quantitative analyses were also performed with glycoproteins having different and relatively fixed oligosaccharide structures from fetuin. Decomposition of neutral sugars would

Table 2. Comparison of the new and regular procedures for quantitative monosaccharides analysis with erythropoietin. The data are the average from 2-5 independent experiments

\begin{tabular}{|c|c|c|}
\hline \multirow{2}{*}{ Sugars } & \multicolumn{2}{|c|}{ Observed (mol sugar/mol protein) } \\
\hline & $\mathrm{New}^{\mathrm{a}}$ & Regularb $^{b}$ \\
\hline Gal & $26.5 \pm 4.1$ & $24.0 \pm 5.5$ \\
\hline Man & $16.2 \pm 3.0$ & $13.2 \pm 3.1$ \\
\hline GlcN & $58.9 \pm 1.0$ & $59.4 \pm 0.9$ \\
\hline Fuc & $3.9 \pm 1.5$ & $4.3 \pm 2.1$ \\
\hline GalN & $4.0 \pm 0.4$ & $3.2 \pm 0.6$ \\
\hline
\end{tabular}

\footnotetext{
${ }^{a}$ The values were produced under the condition of $6 \mathrm{M} \mathrm{HCl}$ at $80^{\circ} \mathrm{C}$ for $2 \mathrm{~h}$.

${ }^{\mathrm{b}}$ The values were produced under the condition of $6 \mathrm{M} \mathrm{HCl}$ for amino and 2 $\mathrm{M}$ TFA for neutral sugars at $100^{\circ} \mathrm{C}$ for $2-4 \mathrm{~h}$, and summed.
}

Table 3. Comparison of experimental and theoretical ratio of monosaccharides in some glycoproteins. The data are the average from three different experiments

\begin{tabular}{ccc}
\hline & $\begin{array}{c}\text { Fibrinogen } \\
\left(\mathrm{Gal}_{2} \mathrm{Man}_{3} \mathrm{GlCNAc}_{4}{ }^{\mathrm{a}}\right)\end{array}$ & $\begin{array}{c}\text { Soybean agglutinin } \\
\left(\mathrm{Man}_{9} \mathrm{GlcNAc}_{2}{ }^{\mathrm{b}}\right)\end{array}$ \\
\hline Gal & $1.8 \pm 0.2$ & 0 \\
Man & $2.5 \pm 0.1$ & $7.6 \pm 0.9$ \\
GlcN & $3.6 \pm 0.3$ & $2.0 \pm 0.1$ \\
\hline
\end{tabular}

Note; The values were based on the results from the hydrolyses with $6 \mathrm{M}$ $\mathrm{HCl}$ at $80^{\circ} \mathrm{C}$ for $2 \mathrm{~h}$.

${ }^{a}$ Taken from Debeire et al. (1985).

${ }^{\mathrm{b}}$ Taken from Dorland et al. (1981).

be reflected sensitively in the molar ratio of neutral sugars to amino sugars when oligosaccharides having more neutral sugars than amino sugars are hydrolyzed. Fibrinogen contains a complex type oligosaccharide, $\mathrm{Gal}_{2^{-}}$ $\mathrm{Man}_{3} \mathrm{GlcNAC}_{4}$ (Debeire et al., 1985) and soybean agglutinin contains a high-mannose type oligosaccharide, $\mathrm{Man}_{9} \mathrm{GlcNAc}_{2}$ (Dorland et al., 1981). When the two glycoproteins were hydrolyzed under the new hydrolysis condition, the ratio between the neutral and amino sugars were $1.8 \pm 0.2: 2.5 \pm 0.1: 3.6 \pm 0.3$ and $0: 7.6 \pm$ $0.9: 2.0 \pm 0.1$ (Gal : Man : GlcN) for fibrinogen and soybean agglutinin, respectively (Table 3 ), which were similar to the theoretical values. These results indicated that the new hydrolysis condition could be used to determine the sugar composition of glycoproteins regardless of oligosaccharide types.

\section{Hydrolysis of a glycoprotein transferred to mem- branes}

To see whether the condition was applicable to blotted proteins, hydrolysis under the new condition was performed with fetuin transferred to PVDF membranes that are widely used for protein immobilization. It showed a relatively low molar ratio of an individual monosaccharide (Gal : Man : GlcN : GalN = $9.5: 6.9$ : $12.1: 2.3$ ), which could be corrected with 1.32 based on $76 \%$ transfer efficiency (Table 1). These small values might be due to transfer efficiency, not yield of hydrolysis. By correcting values, the composition values became 12.5:9.1: $15.9: 3.0$, close to the values obtained from fetuin in solution. It suggested that the condition also could be used for quantitative sugar analysis of PVDF blotted proteins.

\section{Discussion}

The information on monosaccharide composition of glycoproteins has been obtained by two independent acid hydrolyses at high temperature. Thus, this study developed a simple and improved method to analyze amino and neutral monosaccharides of glycoproteins simultaneously. The new hydrolytic condition produced 
very similar compositional ratios to theoretical or experimental values obtained by the commonly used methods from several glycoproteins. It might generate smaller errors than the regular method since it needs only onestep hydrolysis in one-tube. Additionally, it simplify to analyze whole monosaccharides including sialic acid, since the new condition applied the same $\mathrm{HCl}$ and temperature, while the regular methods use two chemicals and different temperatures. We do not exclude possibility of variations between glycoproteins. All the results in this study were at least consistent to suggest that it could be a good alternative for regular procedures to obtain essentially valuable information for monosaccharides composition of glycoproteins. Future studies will focus on generalization of a new condition for analysis of another glycoproteins and minimize errors for monosaccharide determination.

\section{Acknowledgements}

We, authors thank to H. J. Pyo for her excellent technical assistant. This work was supported by grant from the Korea Basic Science Institute.

\section{References}

Debeire, P., Montreuil, J., Moczar, E., van Halbeek, H. and Vliegenthart, J. F. G. (1985) Primary structure of two major glycans of bovine fibrinogen. Eur. J. Biochem. 151: 607-611

Dorland, L., van Halbeek, H., Vliegenthart, J. F. G., Lis, H. and Sharon, N. (1981) Primary structure of the carbohydrate chain of soybean agglutinin. J. Biol. Chem. 256: 7708-7711

Edge, A. S. and Spiro, R. G. (1987) Presence of an Oglycosidically linked hexasaccharide in fetuin. J. Biol. Chem.
262: 16135-16141

Fan, J.-Q., Namaki, Y., Matsuoka, K. and Lee, Y. C. (1994) Comparison of acid hydrolytic conditions for Asn-linked oligosaccharides. Anal. Biochem. 219: 375-378

Green, E. D., Adelt, G., Baenziger, J. U., Wilson, S. and Halbeek, H. V. (1988) The asparagine-linked oligosaccharides on bovine fetuin. Structural analysis of $\mathrm{N}$-glycanase-released oligosaccharides by $500-\mathrm{MHz}{ }^{1} \mathrm{H}$ NMR spectroscopy. J. Biol. Chem. 263: 18253-18268

Ha-Lee, Y. M., Lee, Y., Kim, Y. K. and Sohn, J. (2000) Crosslinking of CD4 induces cytoskeletal association of CD4 and p56 ${ }^{\text {lck }}$. Exp. Mol. Med. 32:18-22

Kornfeld, R. and Kornfeld, S. (1985) Assembly of asparaginelinked oligosaccharides. Annu. Rev. Biochem. 54: 631-664

Lee, D. E., Ha, B. J., Kim, S. J., Park, J. S., Yoo, R. A., Oh, M. S. and Kim, H. S. (1996) Carbohydrate structure of $N$ - and $O$-linked oligosaccharides of human erythropoietin expressed in Chinese hamster ovary cells. J. Biochem. Mol. Biol. 29: 266-271

Spellman, M. W. (1990) Carbohydrate characterization of recombinant glycoproteins of pharmaceutical interest. Anal. Chem. 62: 1714-1722

Spiro, R. G. and Bhoyroo, V. D. (1974) Structure of the Oglycosidically linked carbohydrate units of fetuin. J. Biol. Chem. 249: 5704-5717

Townsend, R. R., Manzi, A., Merkle, R. K., Rohde, M. F., Spellman, M., Smith, A. and Carr, S. A. (1997) Quantitative monosaccharide analysis: A multi-center study. ABRF News 8: $14-21$

Weitzhandler, M., Kadlecek, D., Avdalovic, N., Forte, J. G., Chow, D. and Townsend, R. R. (1993) Monosaccharide and oligosaccharide analysis of proteins transferred to polyvinylidene fluoride membranes after sodium dodecyl sulfatepolyacrylamide gel electrophoresis. J. Biol. Chem. 268: 5121-5130 